\title{
Energetic and economic viability of olive stone recovery as a renewable energy source: a Southern Italy case study
}

\author{
Alessandro Parenti, Piernicola Masella, Lorenzo Guerrini, Antonio Guiso, Paolo Spugnoli \\ Dipartimento Gestione Sistemi Agrari, Alimentari e Forestali, Università di Firenze, Italy
}

\begin{abstract}
The recovery of olive stone for biofuel purpose is becoming increasingly widespread in olive milling plants. The study looks at the economic and energetic benefits of using a de-stoner machine in a smallto-medium sized oil milling plant. The performance of the olive stone recovery system was evaluated over a full olive oil production season. The energetic viability of the de-stoner was assessed using an energy balance. The break-even point was achieved when about 76 tons of olives had been processed, which is less than the annual production of the mill under study. Similarly, in economic terms, the machine covered its costs after about the half of its technical life.
\end{abstract}

\section{Introduction}

Energy generation from biomass is becoming more widespread and gaining in popularity worldwide. Biomass is a generic term for all organic matter produced as a result of photosynthesis, as well as municipal, animal and industrial organic waste. Among these, agricultural by-products are attracting increasing interest (Demirbas, 2005). The review by Rodríguez et al. (2008) showed that the olive oil industry produces significant quantities of by-products, including a valuable lignocellulosic biomass, namely olive stones (OS), which can be used

Correspondence: Lorenzo Guerrini, Dipartimento Gestione Sistemi Agrari, Alimentari e Forestali (GESAAF), Università di Firenze, Piazzale delle Cascine 18, 50144 Firenze, Italy.

Tel.: +39.055.2755932.

E-mail: lorenzo.guerrini@unifi.it

Key words: bioenergy, biomass, olive oil waste, agricultural by-products.

Acknowledgments: the authors would like to thank the Frantoio oleario Giovazzini Giuseppe who made their plant available for our tests, and Pietro Giovazzini for performing the measurements during his doctoral work.

Received for publication: 20 February 2014.

Accepted for publication: 14 June 2014.

(C) Copyright A. Parenti et al., 2014

Licensee PAGEPress, Italy

Journal of Agricultural Engineering 2014; XLV:230

doi:10.4081/jae.2014.230

This article is distributed under the terms of the Creative Commons Attribution Noncommercial License (by-nc 3.0) which permits any noncommercial use, distribution, and reproduction in any medium, provided the original author(s) and source are credited. as biofuel. In fact, based on its combustion performance (Miranda et al., 2008), OS are a viable alternative to wood pellets (a biomass fuel that is experiencing considerable market expansion). According to Cordero et al. (2004), OS are also characterized by low ash content and very low sulphur content that indicate a high-quality fuel.

With regard to olive oil extraction systems, the main by-products of the process are vegetation water and so-called virgin pomace oil (VP). This is a coarse, semi-solid mixture containing residual oils, water and the drupe's solid parts (pulp and woody fragments of the pits) that are left over from the crushing phase. This latter fraction can be separated at various times during processing with differing degrees of efficiency, including: from the olive paste before oil extraction, from the virgin pomace immediately after mechanical oil extraction, and from the exhausted pomace after solvent extraction of the residual oil (Pattara et al., 2010). The amount of VP and OS is highly dependent on the system that is adopted for the extraction of the olive oil. Among the three most widespread extraction techniques (pressing, three and twophase decanter) the amount of VP obtained, thus the amount of waste material generated, varies from $25 \%$ for pressing to $85 \%$ for the twophase decanter technique, by weight of olive drupes mass (Di Giovacchino and Prezioso, 2006). The recovery of OS ranges from 12$20 \%$ of the processed VP mass. Despite this variability, few studies have focused on the energetic and economic viability of OS recovery. This study examines an industrial plant equipped with a three-phase decanter. This configuration was chosen as it is in widespread use and representative of a large number of Italian olive oil mills.

\section{Materials and methods}

\section{Experimental procedure}

The study was carried out at an industrial olive mill in the South of Italy (the Calabria region). The mill operates a continuous threephase horizontal centrifuge [ 4015 CI Plus, Barracane s.r.l., Modugno (BA), Italy], with an actual work rate of about $1000 \mathrm{~kg} \mathrm{~h}^{-1}$ of olive drupes and a yearly running time of $650 \mathrm{~h}^{\text {year }}{ }^{-1}$. The plant was also equipped with a continuous de-stoner machine [5DN/SP, Clemente s.r.l., Flagogna (UD), Italy] that separated OS from VP. This machine uses a cylindrical fixed grid and an inner screw conveyor rotating at $1350 \mathrm{rpm}$ to separate the two materials. The grid (length $0.95 \mathrm{~m}$, internal diameter $0.40 \mathrm{~m}$ ) has two contiguous sections of length 0.2 and 0.7 $\mathrm{m}$, and mesh size of 2.7 and $2.0 \mathrm{~mm}$ respectively. The VP is continuously fed into the grid through a stationary inlet tube. The centrifugal force causes solids to accumulation on the wall of the grid and the extrusion of all materials (mainly pulp) with dimensions smaller than the mesh size. The rotating conveyor continuously removes the solids (mainly OS) that are caught by the grid and moves them towards the discharge outlet. The machine was equipped with three, three-phase alternating-current $(\mathrm{AC})$ motors $(380 \mathrm{~V})$, which were used, respectively, to feed the VP $(0.75 \mathrm{~kW})$, discharge the separated products $(0.75$ 
$\mathrm{kW})$, and move the rotating conveyor $(15 \mathrm{~kW})$. The total installed power was therefore $16.5 \mathrm{~kW}$. The mill's standard working procedure involved batch processing of different varieties of olive drupes, mainly the cultivars grossa di cassano, carolea and tondina in variable proportions. The estimated annual processing capacity of olive drupes was about 650 tons. In order to take into account changes in the physical properties of olives during the harvesting season (October to December), three tests were performed at 15-day intervals. In each test the de-stoner machine was monitored for three consecutive working days. Its electric energy consumption and the flow of mass materials (olive drupes and OS) during the standard OS recovery process were recorded. Moreover, during each trial, an OS sample (one sample per working day) was taken and the moisture content determined.

\section{Analyses}

Electric energy consumption of the de-stoner was recorded by means of a portable electrical network analyser [model Multiver 3SN from Dossena s.n.c., Cavenago d'Adda (LO), Italy].

The moisture content of OS was determined by drying $50 \mathrm{~g}$ samples at a temperature of $105^{\circ} \mathrm{C}$ in a mechanical convection air oven, and calculated from the loss in mass of the samples (Samuelsson et al., 2006).

The energy analysis was performed taking into account the following entries: i) embedded energy of the OS extraction machine (destoner); this energy corresponds to the sum of fossil energy required to produce the machine and the energy required to deliver it to the farm; ii) the conventional energy required producing electricity to run the de-stoner; iii) the thermal energy potentially available from the combustion of OS.

Specific data on the embedded energy of the de-stoner are unavailable in literature, thus data were gathered from Audsley (1997) under the entry named agricultural inputs - other machines. Specifically, Audsley (1997) reports the total fossil energy for the following categories that were summed up to determine the embedded energy of the de-stoner: materials input (48.46 $\left.\mathrm{MJ} \mathrm{kg}^{-1}\right)$, manufacturing (19.61 MJ $\left.\mathrm{kg}^{-1}\right)$, repairs and maintenance (21.63 $\mathrm{MJ} \mathrm{kg}^{-1}$ ), transport to the farm $\left(0.0005377 \mathrm{MJ} \mathrm{kg} \mathrm{km}^{-1}\right)$. Considering a mass of the de-stoner of $850 \mathrm{~kg}$ and a distance of $223 \mathrm{~km}$ between the manufacturer site and the farm under study, the total embedded energy of the de-stoner accounts to 76,347 MJ. The conventional energy required for the de-stoner operation was assessed on the base of the grid electricity consumption recorded during the trials and energy required for the production of electricity itself. The latter was computed from the process of electricity production inventoried in ELCD core II life cycle assessment (LCA) database, named Electricity Mix, consumption mix, at consumer, AC, $1 k V-60 k V I T$ referred to the Italian mix at 2010 . The process dataset was imported (ILCD format) in openLCA and analyzed according to the impact assessment method of cumulative energy demand. The method maps all the energy flows needed to produce $1 \mathrm{MJ}$ of electric energy. The computation gives $2.95 \mathrm{MJ} \mathrm{MJ}_{\mathrm{EL}^{-1}}$ (corresponding to $10.92 \mathrm{MJ}$ $\mathrm{kWh}_{\mathrm{EL}}{ }^{-1}$ ) shared as $75 \%$ fossil sources, 12.5 nuclear sources, and 12.5 renewable sources.

The thermal energy potentially available from the combustion of OS has been estimated assuming a lower heating value (LHV) of $19.2 \mathrm{MJ}$ $\mathrm{kg}^{-1}$ OS dry basis, according to Mata-Sánchez et al. (2014). All the energy computations were referred to the OS dry weigh, based on the average OS moisture content determined during the trials. Furthermore, the thermal energy potentially available from OS was considered irrespective of the thermal conversion devices, thus any conversion efficiency has been considered.

The economic analysis was based on the estimation of the following indicators:

- net present value (NPV):

$$
N P V=\sum_{k=1}^{N} \frac{F_{k}}{(1+i)^{k}}-T C l
$$

where $\mathrm{N}$ is the life of the de-stoner machine, assumed to be 8 years, $i$ is the annual interest rate, assumed to be $6 \%, \mathrm{~F}_{k}$ is the annual flow cash in the $k^{\text {th }}$ year, and TCI is the total capital investment. $\mathrm{F}_{k}$ is calculated as: $F_{k}=R_{O S}-T O C$, where $\mathrm{R}_{0 S}$ is the revenue generated from the sale of OS and TOC is total operating costs;

- profitability index (PI):

$$
P I=\frac{\sum_{k=1}^{N} \frac{F_{k}}{(1+i)^{k}}}{T C l}
$$

- internal rate of return (IRR): defined as the value $i$ that makes the NPV value zero;

- payback time (PBT): defined as the time required recovering the initial investment.

\section{Results and discussion}

Table 1 reports the moisture content of OS sampled during trials. Immediately after separation, the overall average moisture content was of $22.1 \%$ (standard deviation of 2.1). Moisture tends to increase during the harvest season, by about $4 \%$ moving from the first to last test. The recorded values agree with what has been recently reported by MataSánchez et al. (2014), where the limit value of the third quartile of 176 OS samples from Spanish mills was $20.83 \%$, with a strong variability spanning from 5.80 (minimum) to $33.20 \%$ (maximum). However, before it is sold as a fuel, the product usually undergoes pre-treatments such as cleaning or drying processes to allow reducing the moisture content to about 10-12\% (Mata-Sánchez et al., 2014). Figure 1 gives an example of the instantaneous absorbed electrical power during the destoner operation, monitored in one out of the three trials belonging to the first test. It is evident the occurrence of several power peaks, that in some cases differ considerably from the mean value. This was supported by the results of the statistical analysis showed in Table 2, where for the sake of simplicity the statistical outputs refer specifically to data of Figure 1. The same outcome has been found for all the other trials. According to Table 2, the mean adsorbed electrical power had some variability as stated by its standard deviation, that accounts for about $28 \%$ of the mean (variation coefficient). Nevertheless, the range is quite high $(13.34 \mathrm{~kW})$, mirroring the presence of the power peaks (above and below the mean) showed in Figure 1. The $5^{\text {th }}$ and $95^{\text {th }}$ percentiles limits were 3.6 and $11.22 \mathrm{~kW}$, respectively, corresponding to a percentage deviation from the median of $56 \%$ and $+36 \%$ respectively. Outliers detection (Hadi Robust Outlier Detection method) identified 94 cases, i.e. about $14 \%$ of total recorded cases. Of these, 24 cases

Table 1. Moisture content of olive stones. Moisture content $(\%)$ *

\begin{tabular}{lc} 
Test 1 & $20.7 \pm 2.1$ \\
Test 2 & $21.1 \pm 1.6$ \\
\hline Test 3 & $24.5 \pm 1.2$ \\
Average $^{\circ}$ & $22.1 \pm 2.1$ \\
\hline${ }^{*}$ Mean value \pm standard deviation of three independent trials; ${ }^{\circ}$ average value \pm standard deviation of the \\
three test.
\end{tabular}


belong to the $5^{\text {th }}$ percentile (lower than $3.6 \mathrm{~kW}$ ) and 10 belong to the $95^{\text {th }}$ percentile (higher than $11.22 \mathrm{~kW}$ ). As stated above, a similar behaviour was recorded in all tests, with slightly differences in the number and magnitude of power peaks, as well as the average input power. Operating parameters are summarized in Table 3, and the corresponding process performance indicators are shown in Table 4. Mean input power was around $9.9 \mathrm{~kW}$ (standard deviation of $1.14 \mathrm{~kW}$ ), with slightly higher values for the second test. Electric energy consumption averaged $2.02 \mathrm{MJ}$ per $\mathrm{kg}$ of dry OS produced. There was variability between the three tests as stated by a variation coefficient of about $16 \%$, where the electrical energy consumption tended to increase as the season progressed. On average, olive stone yield was $5.84 \mathrm{~kg}$ dry mass (0.12 standard deviation) per $100 \mathrm{~kg}$ of processed drupes. As shown in Figure 1, there were periods of variable length, during which power absorption was minimal. These intervals correspond to dead times, i.e. times when the machine was empty, resulting in energy consumption with no productivity. It is therefore interesting to estimate the effect of these dead times on the total working time of the machine. To this end, we assumed dead times to include all those periods that lasted at least five consecutive minutes, during which the power requirement was less than $4 \mathrm{~kW}$ (i.e. the input power when the de-stoner was empty and representing the minimal electric load). This threshold value was selected according to the outliers detection reported above, where of the 94 outlying cases, 84 cases fall below $4 \mathrm{~kW}$. Of these, 60 cases belong to the $95^{\text {th }}$ percentile being lower than $3.6 \mathrm{~kW}$, and corresponding to about $24 \%$ of total installed power of the de-stoner supporting the importance of these dead time on the overall performance of the machine. As shown in Table 3, dead times accounted for $4.3 \%$ of total energy consumption on average (1.16 standard deviation). Based on the data collected during de-stoner operation, an energy analysis of the OS recovery process was carried out. This evaluated the total quantity of OS to be produced such as the thermal energy potentially available from OS combustion (thermal energy revenue, namely TER), equals (energy break-even point, namely EBP) the total energy required for the recovery process (total energy cost, namely TEC):

$$
\mathrm{EBP}=\text { TER-TEC } ; \mathrm{EPB}=0
$$

The term TEC in equation 3 was computed as the sum of the embedded energy of the de-stoner (fixed energy cost) plus the total operating electrical energy of de-stoner for a given amount of OS to be processed and resulting from the product of OS average specific electrical energy consumption (as MJ kg-1 OS) of Table 4 and the amount of processed OS. The term TER was computed as the product between the OS LHV (19.2 $\mathrm{MJ} \mathrm{kg}^{-1}$ dry weigh) and the produced amount of OS. Both the energy terms TEC and TER were plotted in Figure 2 as a function of increasing amount of produced OS. The EBP, corresponding to the intersection of the two straight lines, resulted of about $4444 \mathrm{~kg}$ of OS to be produced. Assuming an OS recovery yield (dry weigh) of $0.0584 \mathrm{~kg}$ $\mathrm{kg}^{-1}$ of olive mass, about $76,095 \mathrm{~kg}$ of olive have to be processed to reach the EBP. In the case under study, this value was reached in the first year after the de-stoner installation. The economic viability of OS recovery was proved by calculating the NPV, PI, IRR investment and PBT (Table 5). This economic analysis is based on current conditions in the oil mill used in this case study, where the recovered OS was sold.

Some further assumptions were made in order to simplify the analysis: i) in the energy calculation, OS recovery was considered as a separate activity, from an economic point of view, to that of oil production; ii) the cost of the VP used in OS recovery was considered to be null as the mill can use it with no cost; iii) the average selling price of OS was $0.14 € \mathrm{~kg}^{-1}$, assessed by a local market analysis; iv) the by-products of the OS separation process (de-stoned pomace) can be disposed of with no extra costs as waste or soil amendment or sent to the pomace processing plant and generates no revenue; $v$ ) the de-stoner was assumed to have a lifespan of 8 years (the term of the investment); vi) the mean annual OS production was estimated as $48,750 \mathrm{~kg}$, based on a yield of $7.5 \%$ (wet basis) of olive drupes processed; vii) in the NPV computation, TCI corresponds to the purchase cost for the de-stoner machine,

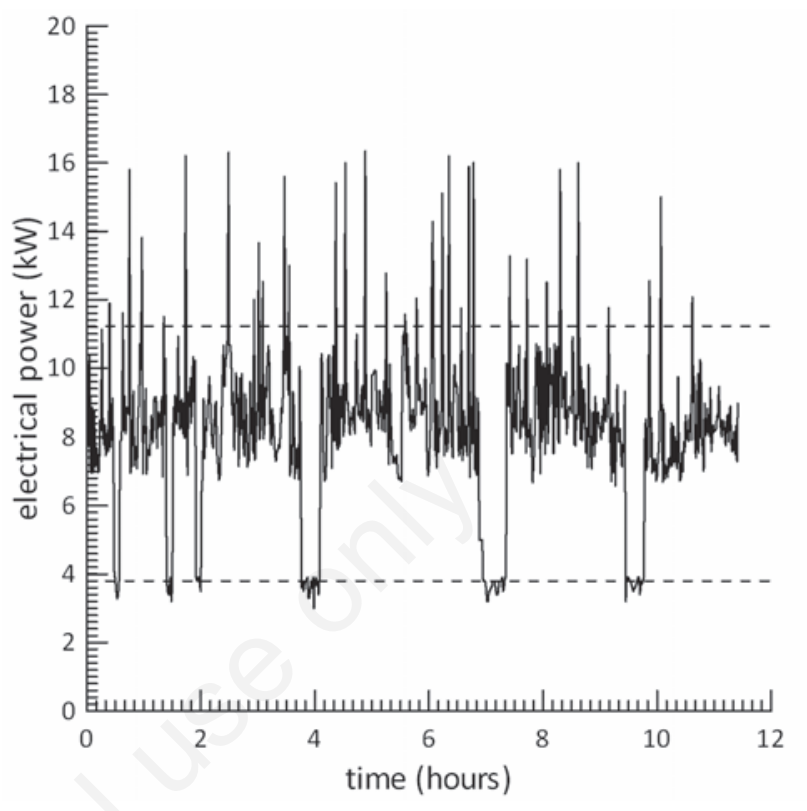

Figure 1. Electrical power adsorbed by the de-stoner over a day during the first test. The dashed lines indicate the limits of the $5^{\text {th }}$ and $95^{\text {th }}$ percentiles.

Table 2. Statistical analysis of instantaneous electric power absorbed during the de-stoner operation.

\begin{tabular}{lc} 
Statistics & Values \\
No. of cases & 685 \\
Minimum (kW) & 3.00 \\
\hline Maximum $(\mathrm{kW})$ & 16.34 \\
Median $(\mathrm{kW})$ & 13.34 \\
\hline Arithmetic mean (kW) & 8.27 \\
Standard error of arithmetic mean & 8.14 \\
\hline $95.0 \%$ lower confidence limit $(\mathrm{kW})$ & 0.09 \\
$95.0 \%$ upper confidence limit $(\mathrm{kW})$ & 7.97 \\
\hline Standard deviation $(\mathrm{kW})$ & 8.31 \\
Coefficient of variation & 2.30 \\
\hline Percentiles (Cleveland method) $(\mathrm{kW})$ & 0.283 \\
$5.00 \%$ & 3.6 \\
\hline $95.00 \%$ & 11.225 \\
\hline
\end{tabular}

Table 3. Operating parameters of the de-stoner.

\begin{tabular}{lcccc} 
& Test 1 & Test 2 & Test 3 & Total \\
Monitoring time (h) & 33.18 & 30.27 & 28.03 & 91.48 \\
Olives (kg) & 29,600 & 30,715 & 22,051 & 82,366 \\
\hline Dry olive stones (kg) & 1697.02 & 1832.058 & 1281.99 & 4811.068 \\
Total electrical energy (MJ)* & 3018.20 & 3469.55 & 3044.75 & 9532.51 \\
\hline Dead time energy (MJ)* & 107.26 & 130.63 & 170.98 & 408.87 \\
\hline *Conversion factor 2.95 ${\text { MJ MJ }{ }^{-1}}^{-1}$ & & & &
\end{tabular}


assumed to be $20,000 €$; viii) TOC correspond to the sum of repairs and maintenance costs (assumed to be $10 \%$ of the cost of the de-stoner machine); insurance costs (assumed to be 1.5\% of the cost of the destoner); electricity costs (estimated on the basis of data collected during monitoring of the de-stoner machine and applying the rates of the Italian Electricity and Gas Authority) equal to $0.0333 € \mathrm{MJ}^{-1}$.

The economic parameters reported in Table 4 confirm the economic viability of olive stone recovery (profitability of investment). Specifically, the PI is higher than 1 and the IRR was significantly higher than the capital cost (assumed to be 6\%). Finally, the PBT corresponds to the $4^{\text {th }}$ working season.

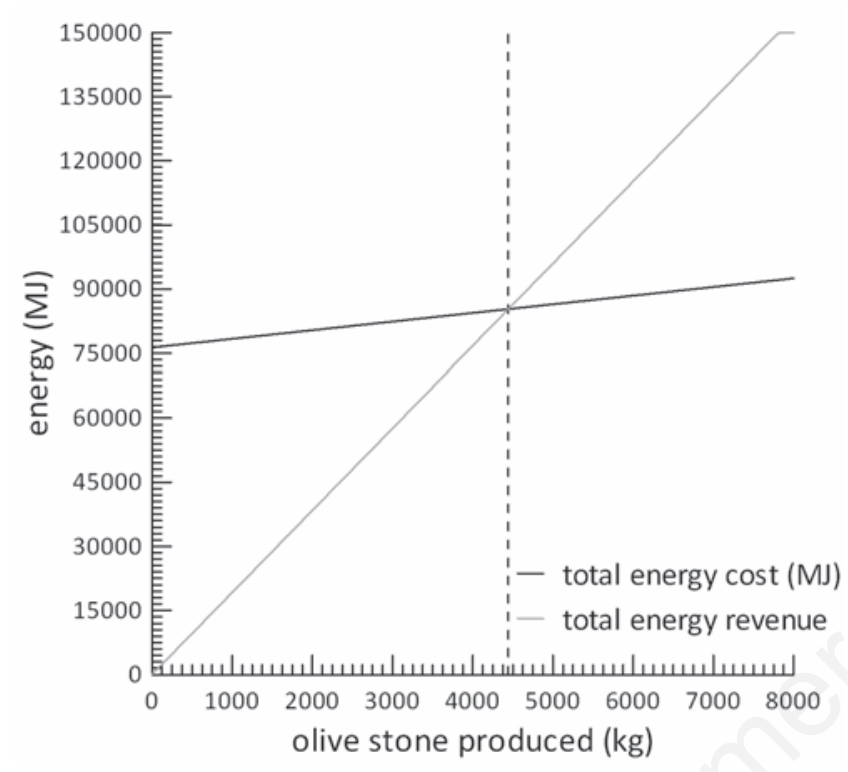

Figure 2. Thermal energy potentially available from olive stones (OS) combustion (total energy revenue) and total energy required for the recovery process (total energy cost) as a function of increasing amount of produced OS.

Table 4. Performance indicators of the de-stoner operation. Olive stones (OS) yield is expressed as the drymass of OS compared to total processed olive mass, and dead time energy is expressed as percentage of total energy consumption.

\begin{tabular}{|c|c|c|c|c|c|}
\hline & Test 1 & Test 2 & Test 3 & Mean & SD \\
\hline Dry OS yield (\% w/w) & 5.73 & 5.96 & 5.81 & 5.84 & 0.12 \\
\hline \multicolumn{6}{|c|}{ Specific electrical energy consumption } \\
\hline$\left(\mathrm{MJ} \mathrm{kg}^{-1} \mathrm{OS}\right)$ & 1.78 & 1.89 & 2.38 & 2.02 & 0.32 \\
\hline Mean input power $(\mathrm{kW})$ & 8.6 & 10.8 & 10.2 & 9.87 & 1.14 \\
\hline Dead time energy (\%) & 3.5 & 3.7 & 5.6 & 4.27 & 1.16 \\
\hline
\end{tabular}

Table 5. Results of economic profitability analysis for olive stones recovery.

\begin{tabular}{lc}
\hline Economic indicator & Value \\
NPV $(€)$ & 15,641 \\
IRR (\%) & 32 \\
\hline PI & 1.78 \\
PBT (years) & 4 \\
\hline NPV, net present value; IRR, internal return rate; PI, profitability index; PBT, pay-back time.
\end{tabular}

In broader terms, given the boundary conditions used here, the economic break-even point is reached after $2600 \mathrm{t}$ of olive oil has been processed and when the de-stoner processes $195 \mathrm{t}$ OS per year.

\section{Conclusions}

Our results clearly confirm the viability of OS recovery in the oil mill under study, both in view of energetic and economic analyses. The framework for our work is typical of the situation for Italian olive mill plants and can be effectively extended to many other olive oil production scenarios. From the energy perspective, the de-stoner covers the cost of OS extraction when about $76 \mathrm{t}$ of olive are processed per year (the equivalent of $4.4 \mathrm{t}$ of dry $\mathrm{OS}$ ). This means that there is a potential energetic advantage for most olive processing plants. From an economic standpoint, although various assumptions had to be made regarding the assessment and importance of the context in which the plant operated, the de-stoner plant began to pay for itself during the fourth season, i.e. about half the assumed plant). Monitoring of the operations of the de-pitting machine showed some variability in electricity consumption, mainly due to variability of the virgin pomace to be processed. Electricity adsorption monitoring also made it possible to evaluate consumption in idle periods, which accounted for about $4.7 \%$ of the total energy consumed. This is an energy loss that could be saved by a proper setting of virgin pomace feeding to the de-stoner. The OS recovery seems to be a major opportunity for the olive oil production sector, and offers a supplementary income for olive mills.

\section{References}

Audsley E., Alber S., Clift R., Cowell S., Crettaz P., Gaillard G., Hausheer J., Jolliet O., Kleijn R., Mortensen B., Pearce D., Roger E., Teulon H., Weidema B., van Zeijts H. 1997. Harmonisation of environmental life cycle assessment for agriculture. Final report, concerted action AIR3CT94-2028. European Commission DG VI, Brussels, Belgium.

Cordero T., Rodrıguez-Mirasol J., Pastrana J., Rodrıguez J. 2004. Improved solid fuels from co-pyrolysis of a high-sulphur content coal and different lignocellulosic wastes. Fuel. 83:1585-90.

Demirbas A. 2005. Biodiesel production from vegetable oils via catalytic and non-catalytic supercritical methanol transesterification methods. Prog. Energ. Combust. 31:466-87.

Di Giovacchino L., Prezioso S. 2006. Utilization of olive mill by-products. pp 379-389. In: Proc. Olivebioteq 2006, Recent advances in the olive industry, Marsala (TP), Italy.

Mata-Sánchez J., Pérez-Jiménez J.A., Díaz-Villanueva M.J., Serrano A., Núñez-Sánchez N., López-Giménez F.J. 2014. Development of olive stone quality system based on biofuel energetic parameters study. Renew. Energ. 66:251-6.

Miranda T., Esteban A., Rojas S., Montero I., Ruiz A. 2008. Combustion analysis of different olive residues. Int. J. Mol. Sci. 9:512-25.

Pattara C., Cappelletti G.M., Cichelli A. 2010. Recovery and use of olive stones: commodity, environmental and economic assessment. Renew. Sust. Energ. Rev. 14:1484-9.

Rodríguez G., Lama A., Rodríguez R., Jiménez A., Guillén R., FernándezBolaños J. 2008. Olive stone an attractive source of bioactive and valuable compounds. Bioresour. Technol. 99:5261-9.

Samuelsson R., Burvall J., Jirjis R. 2006. Comparison of different methods for the determination of moisture content in biomass. Biomass Bioenerg. 30:929-34. 